\title{
Zur Theorie der Narkose.
}

\section{Über den Einfluss der Temperatur auf die Adsorbierbarkeit, das Kolloidfällungsvermögen und die Wirknngsstärke einiger Narkotika.}

\author{
Von.
}

Dr. R. Bierich.

(Aus dem physiologischen Institut der Universität Kiel.)

(Fingegangen am 11. Oltober 1918.)

Die von Hans Horst Meyer und Overton entwickelte Lipoidtheorie der Narkose hat durch den ersteren im Jahre 1901 eine besonders wirksame Stütze mit dem Nachweis erhalten ${ }^{1}$ ), dass, wenu der Verteilungsquotient eines Narkotikums für Öl und Wasser mit der Temperatur steigt, sein Schwellenwert sinkt, dass, wenn er sinkt, sein Schwellenwert steigt. Dies Ergebnis erhielt Me yer bei der Untersuchung der sechs Narkotika: Salicylamid, Benzamid, Monacetin, Äthylalkohol, Chloralhydrat und Aceton, welche sich, entsprechend der folgenden Tabelle, die die Untersuchungsresultate von $\mathrm{H}$. Meyer kurz wiedergibt, in zwei Gruppen von je drei Substanzen gliedern:

\begin{tabular}{|c|c|c|c|c|}
\hline \multirow{3}{*}{ Verbindung } & \multirow{2}{*}{\multicolumn{2}{|c|}{$\begin{array}{c}\begin{array}{c}\text { Schwellenwerte der } \\
\text { narkotischen Wirkung }\end{array} \\
\begin{array}{c}\text { Wirksame Verdünnung } \\
\text { der Normallösungen }\end{array}\end{array}$}} & \multirow{2}{*}{\multicolumn{2}{|c|}{$\begin{array}{l}\text { Verteilungsquotient } \\
\qquad \frac{\text { Oึ1 }}{\text { Wasser }}\end{array}$}} \\
\hline & & & & \\
\hline & bei $3^{\circ} \mathrm{C}$. & bei $30^{\circ} \mathrm{C}$. & bei $3^{\circ} \mathrm{C}$ & ei $30^{\circ} \mathrm{C}$. \\
\hline 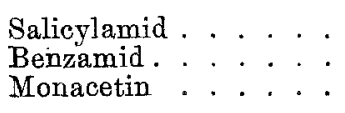 & $\begin{array}{l}1: 1300 \\
1: 500 \\
1: 90\end{array}$ & $\begin{array}{l}1: 600 \\
1: 200 \\
1: 70\end{array}$ & $\begin{array}{l}2,28 \\
0,67 \\
0,093\end{array}$ & $\begin{array}{l}1,40 \\
0,43 \\
0,066\end{array}$ \\
\hline $\begin{array}{l}\text { Athylalkohol . . . . } \\
\text { Chloralhydrat. . . . } \\
\text { Aceton . . . . . . }\end{array}$ & $\begin{array}{l}1: 3 \\
1: 50 \\
1:: 3\end{array}$ & $\begin{array}{l}1: 7 \\
1: 250 \\
1: 7\end{array}$ & $\begin{array}{l}0,024 \\
0,053 \\
0,140\end{array}$ & $\begin{array}{l}0,046 \\
0,236 \\
0,195\end{array}$ \\
\hline
\end{tabular}

Mit diesen interessanten Verhältnissen hat man sich in den 17 seitdem verstrichenen Jahren nicht mehr beschäftigt, insbesondere eine für die Lipoidtheorie gewiss belangreiche Erweiterung des Unter-

1) Hans Meyer, Archiv f. experiment. Pathol. u. Pharm. Bd. 16 S. 338. 1901 . 
suchungsmaterials nicht vorgenommen. Erst während die im folgenden beschriebenen Versuche im Gange waren, erschien eine Arbeit von v. Issekutz ${ }^{1}$ ), welcher sich mit der Frage des Temperatureinflusses auf die Narkotika von neuem beschäftigt und dabei ebenso, wie auch ich, die für die Theorie der Narkose inzwischen erheblich geänderte Sachlage in Rechnung zieht ${ }^{2}$ ).

Denn seither ist erstens durch die Angaben von J. Tra ube ${ }^{3}$ ) u. a. bekannt geworden, dass die narkotische Kraft sich im allgemeinen nicht bloss parallel mit dem Verteilungsquotienten für 001 : Wasser ändert, sondein auch parallel mit der Oberflächenaktivität bzw. Adsorbierbarkeit, und zweitens haben O Warburg und Wiesel ${ }^{4}$ ), Battelli und Stern ${ }^{5}$ ) u. a. gezeigt, dass die Narkotika, ebenfalls entsprechend ihrer narkotischen Kraft, auf alle möglichen Kollside dispersionsvermindernd wirken. Man kann also der Lipoidtheorie eine Adsorptions- und eine Kolloidtheorie der Narkose gegenüberstellen; inwieweit diese beiden innerlich verwandt sind, braucht hier nicht erörtert zu werden.

Diese Sachlage macht es aber notwendig, zur Lipoidtheorie von neuem dadurch Stellung zu nehmen, dass man zusieht, ob Temperaturänderungen die Oberflächenaktivität der Narkotika und ihr Kolloidfällungsvermögen im Sinne ihrer narkotischen Wirksamkeit und entsprechend ihren Verteilungsquotienten nach $\mathrm{H}$. Meyer ändern oder nicht.

v. Issekutz hat in der eitierten Arbeit diese Aufgabe in der Weise in Angriff genommen, dass er stalagmometrisch die Oberflächen: aktivität von Narkotikumlösungen, insbesondere auch von den von H. Meyer bei seinen Temperaturversuchen verwendeten Lösungen bei $6-8^{\circ}$ und bei $33-36^{\circ}$ bestimmte. Er gibt an, dass, genau gemäss den Meyer'schen Angaben über die Temperaturempfindlichkeit der Narkose, bei Salicylamid, Benzamid und Monacetin die Oberflächenaktivität mit der Temperatur abnimmt, während sie bei Äthylalkohol, Chloralhydrat und Aceton steigt. Die angestrebte Entscheidung zugunsten oder zuungunsten der Lipoidtheorie würde danach nicht zu

1) v. Issekutz, Biochem. Zeitschr. Bd. 88 S. 213.1918.

2) Anmerkung bei der Korrektur: Nach Absendung des Manuskriptes erhielt ich Kenntnis von einer kürzlich erschienenen Arbeit von R. Unger (Birchem. Zeitschr. 89, 238. 1918), in welcher das gleiche Thema behandelt wird, wie in meiner Abhandlung und in der anschliessenden von $\mathrm{Höb}$ er. Tch beschränke mich hier auf den Hinweis, dass in den wesentlichen Punkten zwischen Unger und uns Übereinstimmung herrscht.

3) J. Traube.' P flüger's Ach. Bd, 123. 419. 1908. Verh. d. deutsch. physik. Ges. Bd. 10,880. 1909.

4) O. Warburg u. Wiesel. Pflüger's Arch. Bd. 144. 465.. 1912.

5) Batelli u. Stern. Bioch. Zeitschr., Bd. 52. 226, 253. 1913. 
treffen sein, da die Oberflächenaktivitäten den Verteilungsquotienten entsprechend temperaturvariabel sind.

Sieht man sich die Zahlen von v. Issekutz aber näher an, so wird man zweifelhaft, ob dieser Schluss genügend gesichert ist. Die Tropfenzahl für Wasser gleich 100 gesetzt, sind zum Beispiel die Tropfenzahlen für $\frac{\text { in }}{60}$ - Salicylamid bei der niederen und der hohen Temperatur 101,86 und 101,0, für $\frac{\mathrm{m}}{50}$ Benzamid 101,32 und 100,94, für $\frac{m}{70}$ Monacetin 101,7 und 101,0. Da das benutzte Stalagmometer mit Wasser in Wirklichkeit einige 30 Tropfen gab, so betrug also der Unterschied bei den beiden Temperaturen immer nur einige Zehntel Tropfen, ist also fast gleich Null. Vielleicht ist er sogar nur durch Zufall vorgetäuscht; denn da bei Salicylamid für die steigenden Konzentrationen $\frac{\mathrm{m}}{1300}, \frac{\mathrm{m}}{600}, \frac{\mathrm{m}}{60}, \frac{\mathrm{m}}{30}$ die Werte $101,1,101,9,101,86(!)$, 100,6 (!) angegeben werden, so sind die Messungen doch offenbar mit im Ergebnis nicht zu vernachlässigenden Fehlern behaftet. Auch die Unterschiec $\mathrm{e}$ in den Tropfenzahlen bei den Lösungen von Äthylalkohol, Chloralhydrat und Aceton, welche die entgegengesetzte Richtung haben, sind ausserordentlich klein; da diese drei Narkotika im Gegensatz zu den ersten drei beim Abtropfen in der höheren Temperatur nachweislich zum Teil verdampfen, so wird vom Verfasser eine Korrektur der beobachteten Zahlen vorgenommen, welche sich meines Erachtens theoretisch nicht begründen lässt.

Meine eigenen Untersuchungen umfassen erstens den Einfluss der Temperatur auf die Adsorbierbarkeit einiger Narkotika an Tierkohle und auf ihre Fähigkeit, Kolloide auszuflocken, und zweitens bestehen sie in einer Nachprüfung der alten Angaben von H. Meyer. Für Anregung und Förderung dieser Versuche exlaube ich mir, auch an dieser Stelle, Herm Prof. Höber zu danken.

\section{Der Einfluss der Temperatur auf die Adsorbierbarkeit einiger Narkotika durch Tierkohle.}

Über den Einfluss der Temperatur auf die Adsorbierbarkeit liegen nur verhältnismässig spärliche Angaben vor. Freundlich bezeichnet nach eigenen Versuchen den Einfluss auf die Adsorbierbarkeit von Essigsäure, Bernsteinsäure, Pikrinsäure an Kohle als sehr gering und gibt einige Daten anderer Autoren an, welche dasselbe besagen ${ }^{1}$ ).

1) Freundlich, Zeitschr. f. physikal. Chemie Bd. 57 S. 386. 1907; Kapillarchemie 1909 S. 169. 
Dementsprechend fand auch ich keine oder nur eine geringfügige Veränderung und diese meist im Sinne einer Abnahme.

Die Versuche wurden mit säuregereinigter Tierkohle (Carbo animalis purissimus Merck) ausgeführt. Je $50 \mathrm{ccm}$ der Narkotikumlösungen wurder durchschnittlich 2 Stunden bei ungefähr $0^{0}$ und $50^{\circ}$ mit der gleichen Menge Kohle in Flaschen mit Glasstopfen geschüttelt, darauf sofort abgesaugt und der Gehalt zum Teil stalagmometrisch bei Zimmertemperatur, und wo dies wegen der Geringfügigkeit der Oberflächenspannungsänderung nicht genau genug war, mit dem Kjeldahlverfahren bestimmt. Der Wasserwert des verwendeten Stalagmometers betrug bei $15^{0} 17,1$ Tropfen.

\section{Versuch 1. Isoamylurethan.}

a) $0,5 \%$.

Tropfenzahl bei $15,5^{0} \quad 28,75$

$50 \mathrm{ccm}+\mathrm{I} \mathrm{g}$ Kohle bei $0^{0} 1 \mathrm{St}$. geschüttelt. " ", $15,5^{0} \quad 18,4$

$50,+1, \quad, \quad, \quad 0^{0} 3,, \quad, \quad, \quad, 15,5^{0} 18,55$

$50,+1, \quad, \quad, 50^{0} 1, \quad, \quad, \quad, \quad, \quad 15,5^{0} 19,25$

$50,+1, \quad, \quad, 50^{\circ} 3, \quad, \quad, \quad, \quad, 15,5^{0} 19,3$

b) $0,25 \%$.

Tropfenzahl bei $15,7^{0} \quad 25,58$

$50 \mathrm{~cm}+1 \mathrm{~g}$ Kohle bei $0^{0} \mathrm{I}$ St. geschüttelt. $\quad$ " , $15,7^{0} \quad 17,6$

$50,+1,, \quad, \quad 0^{0} 3, \% \quad, \quad, \quad, \quad, 15,7^{0} 17,8$

$50, \quad+1, \quad, \quad, 50^{\circ} 1, \quad, \quad, \quad, \quad, 15,7^{0} \quad 17,9$

$50,+1, \quad, \quad$, $50^{\circ} 3, \quad \quad, \quad, \quad, 15,7^{\circ} 17,9$

Versuch 2. Isobutylurethan.

$1 \%$.

Tropfenzahl bei $16,5^{0} \quad 25,3$

$50 \mathrm{ccm}+1 \mathrm{~g}$ Kohle bei $0^{0} 1 \mathrm{St}$. geschüttelt. , , , $16,0^{\circ} 21,6$

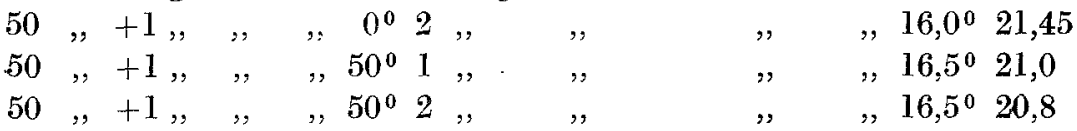

Versuch 3. Heptylalkohol.

$0,058 \%$.

Tropfenzahl bei $15,0^{\circ} 25,0$

$50 \mathrm{ccm}+1 \mathrm{~g}$ Kohle bei $0^{0} 2$ St. geschüttelt. , , , $15,0^{\circ} \quad 17,82$ $50 \quad,+1 ", \quad, 50^{\circ} 2, " \quad, \quad, \quad, 15,0^{0} \quad 17,7$

Bei den Lösungen von Phenylharnstoff, Benzamid und Salicylamid war die Tropfenzahl von der des Wassers nur so wenig verschieden dass ihre Konzentration sich genauer erst nach $\mathrm{Kjeldahl}$ bestimmen liess.

Versuch 4. Phenylharnstoff. $0,4 \%$.

$50 \mathrm{ccm}+0,25 \mathrm{~g}$ Kohle bei $0^{0} 2$ St. geschüttelt. Adsorbiert $50 \%$

$50, \quad+0,25, \quad, \quad, 50^{\circ} 2, \quad, \quad, \quad 46,25 \%$ 
Versuch 5. Salicylamid. $0,2 \%$.

$50 \mathrm{ccm}+0,25 \mathrm{~g}$ Kohle bei $0^{0} 2 \mathrm{St}$. geschüttelt. Adsorbiert $83,7 \%$ $50,+0,25 \%, \quad, 500^{\circ} 2, \quad " \quad, \quad 81,2 \%$

Versuch 6. Benzamid. 0,8\%.

$50 \mathrm{ccm}+0.25 \mathrm{~g}$ Kohle bei $0^{0} 2 \mathrm{St}$. geschüttelt. Adsorbiert $25,3 \%$ $50, \quad+0,25, \quad, \quad, 500^{0} 2, \quad, \quad, \quad 25,3 \%$

Nach diesen Versuchen ist die Wirkung der Temperatur auf die Adsorption der Narkotika so unbedeutend, dass es nicht angängig ist, daraus für die Abhängigkeit der Narkose von der Temperatur etwas zu folgern.

\section{Der Einfluss der 'Temperatur anf das Kolloidfällungs- vermögen einiger Narkotika.}

Es ist bereits auf die Versuche von 0 . Warburg und Wiesel und von Battelli und Stern kurz hingewiesen worden, in denen gezeigt wurde, dass in Hefepresssaft und in Lösungen von Nukleoproteiden durch Narkotika Flockungen zu erzeugen sind, welche im allgemeinen um so dichter sind, je grösser die narkotische Kraft des Fällungsmittels ist. Neuerdings haben Freundlich und Rona ${ }^{1}$ ) festgestellt, dass diese Fähigkeit von Nichtelektrolyten, wie es die Narkotika sind, die Beständigkeit eines Sols aufzuheben, an die Gegenwart von Elektrolyten gebunden ist; zu ihrem Nachweis bedienten sie sich des Eisenhydroxydsols. Die von ihnen ausgesprochene Vermutung, dass auch die von Warburg und Wiesel und von Battelli und Stern beobachteten Flockungen nur durch die Beimischung von anorganischen Salzen zu den organischen Kolloiden bedingt seien, ist neuerdings in diesem Institut von 0 . Meyerhof ${ }^{2}$ ) als zutreffend erwiesen worden.

Meine Untersuchungen bezweckten nun die Prüfung, ob Temperaturerhöhung das Koagulationsvermögen der Narkotika verändert, speziell ob bei manchen Narkoticis der Einfluss anders gerichtet ist als bei anderen.

Die eine meiner Versuchsreihen betrifft, in Anlehnung an die Untersuchungen von Freundlich und Rona, das Eisenhydroxydsol. Ferrum oxydatum dialysatum liquidum $10 \%$ duplex von Merck wurde zu möglichst vollständiger Entfernung des $\mathrm{Cl}-8$ Tage lang dialysiert. Sodann wurde, genau so wie Freundlich und Rona es angeben, zu 4,5 ccm des Sols $50 \mathrm{~cm}$ der Narkotikumlösung oder, bei den Kontrollproben, Wasser hinzugefügt. Von diesen Gemischen wurden je $5 \mathrm{ccm}$ mit je $1 \mathrm{ccm}$ Kochsalzlösung von verschiedener

1) Freundlich und Rona, Biochem. Zeitschr. Bd. 81 S. 87. 1917.

2) O. Meyerhof, ebenda Bd. 86 S. 325. 1918. 
Konzentration versetzt und gleiche Proben sodann bei $0^{0}$ und bei $50^{\circ}$ stehengelassen.

Die folgenden Tabellen geben die Ergebnisse wieder. Die Anzahl der Pluszeichen bedeutet ein Maass für die Stärke der Flockung.

Versuch 1. Äthylurethan. 17,8\%.

\begin{tabular}{|c|c|c|c|c|c|c|}
\hline $\begin{array}{l}\text { Millimol } \mathrm{NaCl} \\
\text { pro Liter }\end{array}$ & 200 & 100 & 50 & 25 & 12,5 & 6,25 \\
\hline $\begin{array}{r}50^{\circ} \mathrm{C} .10^{\prime} \\
30^{\prime} \\
60^{\prime} \\
100^{\prime}\end{array}$ & $\begin{array}{l}t+t \\
+t+ \\
+t+ \\
+t+\end{array}$ & $\begin{array}{l}+ \\
+t \\
+t+ \\
+t+\end{array}$ & $\begin{array}{c}0 \\
\text { Hauch } \\
+ \\
+\end{array}$ & $\begin{array}{c}0 \\
\text { Hauch } \\
0 \\
0\end{array}$ & $\begin{array}{l}0 \\
0 \\
0 \\
0\end{array}$ & $\begin{array}{l}0 \\
0 \\
0 \\
0\end{array}$ \\
\hline $\begin{array}{r}0^{\circ} \text { C. } 10^{\prime} \\
30^{\prime} \\
60^{\prime} \\
100^{\prime}\end{array}$ & $\begin{array}{l}t+ \\
+t+ \\
+t+ \\
t+t\end{array}$ & $\begin{array}{l}t \\
+ \\
t+ \\
t+\end{array}$ & $\begin{array}{l}0 \\
0 \\
0 \\
0\end{array}$ & $\begin{array}{l}0 \\
0 \\
0 \\
0\end{array}$ & $\begin{array}{l}0 \\
0 \\
0 \\
0\end{array}$ & $\begin{array}{l}0 \\
0 \\
0 \\
0\end{array}$ \\
\hline
\end{tabular}

Versuch 2. Amylurethan. 0,5\%.

\begin{tabular}{|c|c|c|c|c|c|c|}
\hline $\begin{array}{l}\text { Millimol NaCl } \\
\text { pro Litex }\end{array}$ & 200 & 100 & 50 & 25. & 12,5 & 6,25 \\
\hline $\begin{array}{r}50^{\circ} \text { C. } 10^{\prime} \\
30^{\prime} \\
60^{\prime} \\
100^{\prime}\end{array}$ & $\begin{array}{l}+t+ \\
++t \\
t+t \\
+t+\end{array}$ & $\begin{array}{l}t+ \\
+t+ \\
+t+ \\
t+t\end{array}$ & $\begin{array}{l}0 \\
+? \\
+ \\
++\end{array}$ & $\begin{array}{l}0 \\
0 \\
+? \\
+?\end{array}$ & $\begin{array}{l}0 \\
0 \\
0 \\
0\end{array}$ & $\begin{array}{l}0 \\
0 \\
0 \\
0\end{array}$ \\
\hline $\begin{array}{r}0^{\circ} \mathrm{C} .10^{\prime} \\
30^{\prime} \\
60^{\prime} \\
100^{\prime}\end{array}$ & $\begin{array}{l}++ \\
++t \\
++t \\
+t+\end{array}$ & $\begin{array}{l}t \\
t+ \\
t+ \\
t+\end{array}$ & $\begin{array}{l}0 \\
+? \\
+? \\
+\end{array}$ & $\begin{array}{l}0 \\
0 \\
0 \\
0\end{array}$ & $\begin{array}{l}0 \\
0 \\
0 \\
0\end{array}$ & $\begin{array}{l}0 \\
0 \\
0 \\
0\end{array}$ \\
\hline
\end{tabular}

Versuch 3. Thymol, gesättigt.

\begin{tabular}{|c|c|c|c|c|c|c|}
\hline $\begin{array}{l}\text { Millimol } \mathrm{NaCl} \\
\text { pro Liter }\end{array}$ & 200 & 150 & 100 & 75 & 50 & 25 \\
\hline $\begin{array}{r}50^{\circ} \mathrm{C} .10^{\prime} \\
30^{\prime} \\
60^{\prime} \\
180^{\circ}\end{array}$ & $\begin{array}{l}+++ \\
+++ \\
+++ \\
t+t\end{array}$ & $\begin{array}{l}+t+ \\
+t+ \\
++t \\
t+t\end{array}$ & $\begin{array}{l}+ \\
+ \\
++ \\
++\end{array}$ & $\begin{array}{c}\text { Hauch } \\
\text { Hauch } \\
+ \\
+\end{array}$ & $\begin{array}{l}0 \\
0 \\
0 \\
0\end{array}$ & $\begin{array}{l}0 \\
0 \\
0 \\
0\end{array}$ \\
\hline $\begin{array}{r}0^{\circ} \mathrm{C} .10^{\prime} \\
30^{\prime} \\
60^{\prime} \\
180^{\prime}\end{array}$ & $\begin{array}{l}+t+ \\
+t+ \\
+t+ \\
t+t\end{array}$ & $\begin{array}{l}+t \\
+t \\
+t \\
+t+\end{array}$ & $\begin{array}{l}t \\
+ \\
+ \\
t+\end{array}$ & $\begin{array}{l}0 \\
0 \\
0 \\
0\end{array}$ & $\begin{array}{l}0 \\
0 \\
0 \\
0\end{array}$ & $\begin{array}{l}0 \\
0 \\
0 \\
0\end{array}$ \\
\hline
\end{tabular}


Versuch 4. Benzamid. $1 \%$.

\begin{tabular}{|c|c|c|c|c|c|c|}
\hline $\begin{array}{l}\text { Millimol } \mathrm{NaCl} \\
\text { pro Liter }\end{array}$ & 200 & 150 & 100 & 75 & .50 & 25 \\
\hline $\begin{array}{r}50^{\circ} \mathrm{C} .10^{\prime} \\
30^{\prime} \\
60^{\prime} \\
120^{\prime}\end{array}$ & $\begin{array}{l}t+t \\
t+t \\
t+t \\
t+t\end{array}$ & $\begin{array}{l}t+ \\
t+ \\
t+t \\
+t+\end{array}$ & $\begin{array}{l}+ \\
+ \\
+ \\
+t\end{array}$ & $\begin{array}{l}0 \\
0 \\
0 \\
+\end{array}$ & $\begin{array}{l}0 \\
0 \\
0 \\
0\end{array}$ & $\begin{array}{l}0 \\
0 \\
0 \\
0\end{array}$ \\
\hline $\begin{array}{r}0^{\circ} \mathrm{O} .10^{\prime} \\
30^{\prime} \\
60^{\prime} \\
120^{\circ}\end{array}$ & $\begin{array}{l}t+ \\
+t+ \\
+t+ \\
+t+\end{array}$ & $\begin{array}{l}t \\
t \\
t+ \\
t+\end{array}$ & $\begin{array}{l}0 \\
0 \\
0 \\
0\end{array}$ & $\begin{array}{l}0 \\
0 \\
0 \\
0\end{array}$ & $\begin{array}{l}0 \\
0 \\
0 \\
0\end{array}$ & $\begin{array}{l}0 \\
0 \\
0 \\
0\end{array}$ \\
\hline
\end{tabular}

Kontroll-Versuch 5. Wasser (zu Versuch 1 u. 2).

\begin{tabular}{c|c|c|c|c|c}
\hline $\begin{array}{c}\text { Millimol NaCl } \\
\text { pro Liter }\end{array}$ & 200 & 100 & 50 & 25 & 12,5 \\
\hline $\mathbf{5 0}^{\circ} \mathrm{C} .10^{\prime}$ & ++ & 0 & 0 & 0 & 0 \\
$30^{\prime}$ & +++ & 0 & 0 & 0 & 0 \\
$60^{\prime}$ & +++ & + & 0 & 0 & 0 \\
$180^{\prime}$ & +++ & ++ & 0 & 0 & 0 \\
\hline \multirow{2}{*}{$\mathbf{0}^{\circ} \mathrm{C} .10^{\prime}$} & ++ & 0 & 0 & 0 & 0 \\
$30^{\prime}$ & ++ & 0 & 0 & 0 & 0 \\
$60^{\prime}$ & ++ & 0 & 0 & 0 & 0 \\
$180^{\prime}$ & ++ & + & 0 & 0 & 0
\end{tabular}

Kontroll-Versuch 6. Wasser (zu Versuch 3 u. 4).

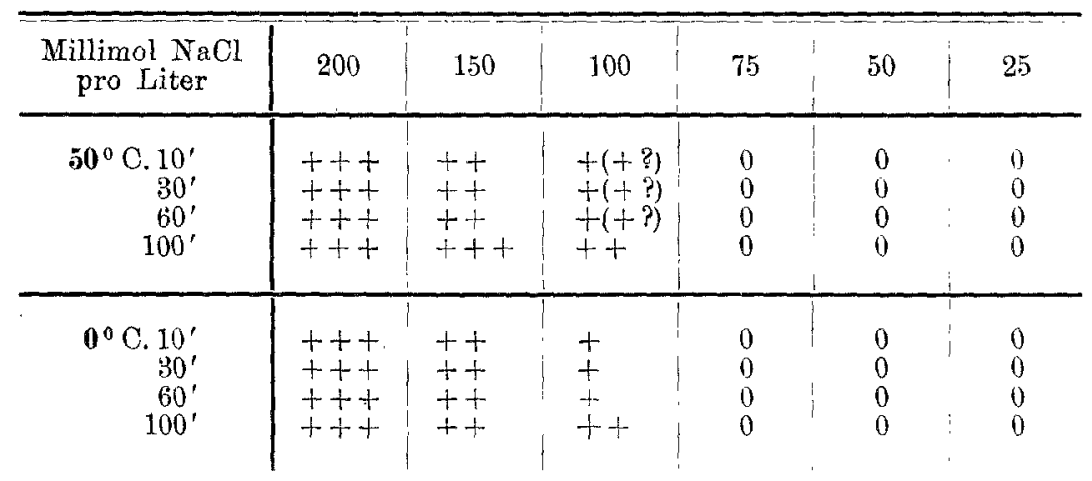

Eine Durchsicht der Tabellen belehrt darüber, dass die vier geprüften Narkotika Äthylurethan, Amylurethan, Thymol und Benzamid, von denen eines, das Benzamid, nach Meyer bei 
höherer Temperatur schwächer narkotisch wirkt als bei niederer, sämtlich die Kochsalzfällung des Eisenhydroxydsols bei höherer Temperatur mehr verstärken als bei niederer; der Unterschied ist freilich nur gering. Die Vermutung, dass vielleicht auch ohne die Gegenwart von Narkotikum die Fällbarkeit des Eisens durch die Wärme begünstigt werde, trifft zwar, wie die Kontrollversuche 5 und 6 lehren, zu, aber in Anwesenheit der Narkotika ist der Unterschied bei den beiden Temperaturen deutlich stärker.

In einer zweiten Versuchsreihe wurde das Ausflocken von denaturiertem Serumalbumin untersucht. Rinderserum wurde mit dem gleichen Volumen konzentrierter Ammonsulfatlösung gefällt, das Filtrat durch 10tägiges Dialysieren gegen Wasser in Pergamentschläuchen von dem Fällungsmittel befreit und so weit verdünnt $(1: 2)$, dass es bei vorsichtigem Erwärmen auf $80-90^{\circ}$ nicht ausflockte.

Von diesem Sol wurde je $1 \mathrm{ccm}$ mit ausprobierten Narkotikummengen und mit $1-3$ Tropfen einer $\frac{m}{100}-$ Kobaltchlorürlösung - bzw. in den Kontrollversuchen mit ebensoviel Wasser - versetzt und dann 1-2 Stunden lang bei $0^{\circ}$ und bei $50^{\circ}$ beobachtet. In Vorversuchen war festgestellt, dass ein Elektrolyt mit mehrwertigem Kation schärfere Unterschiede ergiebt als Kochsalz.

Uber die Ergebnisse unterrichten die folgenden Tabellen:

Verauch 1. Isobutylalkohol. $10 \%$.

\begin{tabular}{|c|c|c|c|c|c|c|}
\hline & $\begin{array}{l}5 \text { Tropfen } \\
\text { Isobutyl- } \\
\text { alkohol, } \\
1 \text { Tropfen } \\
\text { Co }\end{array}$ & $\begin{array}{l}\text { 4 Tropfen } \\
\text { Isobutyl- } \\
\text { alkohol, } \\
\text { 2 Tropfen } \\
\text { Co }\end{array}$ & $\begin{array}{l}4 \text { Tropfen } \\
\text { Isobutyl- } \\
\text { alkohol, } \\
3 \text { Tropfen } \\
\text { Co }\end{array}$ & $\begin{array}{c}5 \text { Tropfen } \\
\text { Wasser, } \\
1 \text { Tropfen } \\
\text { Co }\end{array}$ & $\begin{array}{c}4 \text { Tropfen } \\
\text { Wasser, } \\
2 \text { Tropfen } \\
\text { Co }\end{array}$ & $\begin{array}{c}4 \text { Tropfen } \\
\text { Wasser, } \\
3 \text { Tropfen } \\
\text { Co }\end{array}$ \\
\hline \multirow[t]{3}{*}{$50^{\circ} \mathrm{C} .10^{\prime}$} & $\begin{array}{c}\text { Grobe } \\
\text { Flockung. }\end{array}$ & $\begin{array}{c}\text { Opales- } \\
\text { cenz. }\end{array}$ & $\begin{array}{l}\text { Feine } \\
\text { Flockung. }\end{array}$ & $\begin{array}{c}\text { Geringe } \\
\text { Opales- } \\
\text { cenz. }\end{array}$ & $\begin{array}{l}\text { Opales- } \\
\text { cenz. }\end{array}$ & $\begin{array}{c}\text { Starke } \\
\text { Opales- } \\
\text { cenz. }\end{array}$ \\
\hline & $\begin{array}{c}\text { Grobe } \\
\text { Flockung } \\
\text { mit Boden- } \\
\text { satz. }\end{array}$ & $\begin{array}{c}\text { Stärkere } \\
\text { Opales- } \\
\text { cenz. }\end{array}$ & $\begin{array}{c}\text { Grobe } \\
\text { Flockung } \\
\text { mit Boden- } \\
\text { satz. }\end{array}$ & $\begin{array}{c}\text { Geringe } \\
\text { Opales- } \\
\text { cenz. }\end{array}$ & $\begin{array}{c}\text { Opales- } \\
\text { cenz. }\end{array}$ & $\begin{array}{c}\text { Starke } \\
\text { Opales. } \\
\text { cenz. }\end{array}$ \\
\hline & $\begin{array}{c}\text { Grobe } \\
\text { Flockung } \\
\text { mit Boden- } \\
\text { satz. }\end{array}$ & Trübung. & $\begin{array}{c}\text { Grobe } \\
\text { Flockung } \\
\text { mit Boden- } \\
\text { satz. }\end{array}$ & $\begin{array}{c}\text { Geringe } \\
\text { Opales- } \\
\text { Cenz. }\end{array}$ & $\begin{array}{c}\text { Opales- } \\
\text { cenz. }\end{array}$ & $\begin{array}{c}\text { Starke } \\
\text { Opales- } \\
\text { cenz. }\end{array}$ \\
\hline $\begin{array}{r}0^{\circ} \mathrm{C} .10^{\prime} \\
30^{\prime} \\
120^{\prime}\end{array}$ & $\begin{array}{l}0 \\
0 \\
0\end{array}$ & $\begin{array}{l}0 \\
0 \\
0\end{array}$ & $\begin{array}{l}0 \\
0 \\
0\end{array}$ & $\begin{array}{l}0 \\
0 \\
0\end{array}$ & $\begin{array}{l}0 \\
0 \\
0\end{array}$ & $\begin{array}{l}0 \\
0 \\
0\end{array}$ \\
\hline
\end{tabular}


Versuch 2. Äthylurethan. $100 \%$.

\begin{tabular}{|c|c|c|c|}
\hline & $\begin{array}{c}20 \text { Tropfen Äthylurethan, } \\
1 \text { Tropfen Co }\end{array}$ & \multicolumn{2}{|c|}{$\begin{array}{l}20 \text { Tropfen Wasser, } \\
1 \text { Tropfen Co }\end{array}$} \\
\hline $\begin{array}{r}50^{\circ} \mathrm{C} .10^{\prime} \\
30^{\prime} \\
120^{\prime}\end{array}$ & $\begin{array}{l}\text { Starke Trübung. } \\
\text { Flockung. } \\
\quad "\end{array}$ & $\begin{array}{c}\text { Schwache } \\
" \\
"\end{array}$ & $\begin{array}{c}\text { Opalescenz. } \\
" \\
"\end{array}$ \\
\hline $\begin{array}{r}0^{\circ} \text { C. } 10^{\circ} \\
30^{\prime} \\
120^{\prime}\end{array}$ & $\begin{array}{c}0 \\
\text { Fast klar. } \\
\text { Fast klar. }\end{array}$ & & $\begin{array}{l}0 \\
0 \\
0\end{array}$ \\
\hline
\end{tabular}

Versuch 3. Propylurethan, $0,5 \%$, direkt im Eiweisssol bei $46^{\circ}$ gelöst.

\begin{tabular}{|c|c|c|c|c|}
\hline & 1 Tropfen Co & 2 Tropfen Co & $\begin{array}{l}\text { Ohne } \\
\text { Propylurethan, } \\
1 \text { Tropfen Co }\end{array}$ & $\begin{array}{l}\text { Ohne } \\
\text { Propylurethan, } \\
2 \text { Tropfen Co }\end{array}$ \\
\hline \multirow[t]{3}{*}{$50^{\circ}$ C. $10^{\prime}\{$} & $\begin{array}{c}\text { Geringe } \\
\text { Opalescenz. }\end{array}$ & $\begin{array}{l}\text { Grobe } \\
\text { Flockung. }\end{array}$ & 0 & $\begin{array}{l}\text { Schwache } \\
\text { Opalescenz. }\end{array}$ \\
\hline & $\begin{array}{c}\text { Etwas stärkere } \\
\text { Opalescenz. }\end{array}$ & $\begin{array}{l}\text { Grobe } \\
\text { Flockung. }\end{array}$ & 0 & $\begin{array}{l}\text { Schwache } \\
\text { Opalescenz. }\end{array}$ \\
\hline & $\begin{array}{c}\text { Etwas stärkere } \\
\text { Opalescenz. }\end{array}$ & $\begin{array}{l}\text { Grobe } \\
\text { Flockung. }\end{array}$ & 0 & $\begin{array}{l}\text { Schwache } \\
\text { Opalescenz. }\end{array}$ \\
\hline $\begin{array}{l}0^{\circ} \text { C. } 10^{\prime} \\
30^{\prime} \\
60^{\prime}\end{array}$ & $\begin{array}{l}0 \\
0 \\
0\end{array}$ & $\begin{array}{l}0 \\
0 \\
0\end{array}$ & $\begin{array}{l}0 \\
0 \\
0\end{array}$ & $\begin{array}{l}0 \\
0 \\
0\end{array}$ \\
\hline
\end{tabular}

Versuch 4. Benzamid, 1\%, direkt im Eiweisssol bei $40^{\circ}$ gelöst.

\begin{tabular}{r|c|c|c|c|c|c}
\hline & $\begin{array}{c}1 \text { Tropfen } \\
\text { Co }\end{array}$ & $\begin{array}{c}2 \text { Tropfen } \\
\text { Co }\end{array}$ & $\begin{array}{c}\text { Tropfen } \\
\text { Co }\end{array}$ & $\begin{array}{c}\text { Ohne } \\
\text { Benzamid, } \\
1 \\
\text { Tropfen } \\
\text { Co }\end{array}$ & $\begin{array}{c}\text { Ohne } \\
\text { Benzamid, } \\
2 \text { Tropfen } \\
\text { Co }\end{array}$ & $\begin{array}{c}\text { Ohne } \\
\text { Benzamid. } \\
3 \text { Tropfen } \\
\text { Co }\end{array}$ \\
\hline $30^{\circ} \mathrm{C.10}$
\end{tabular}


Versuch 5. Salicylamid, 0,05\%, direkt im Eiweisssol bei $40^{\circ}$ gelöst.

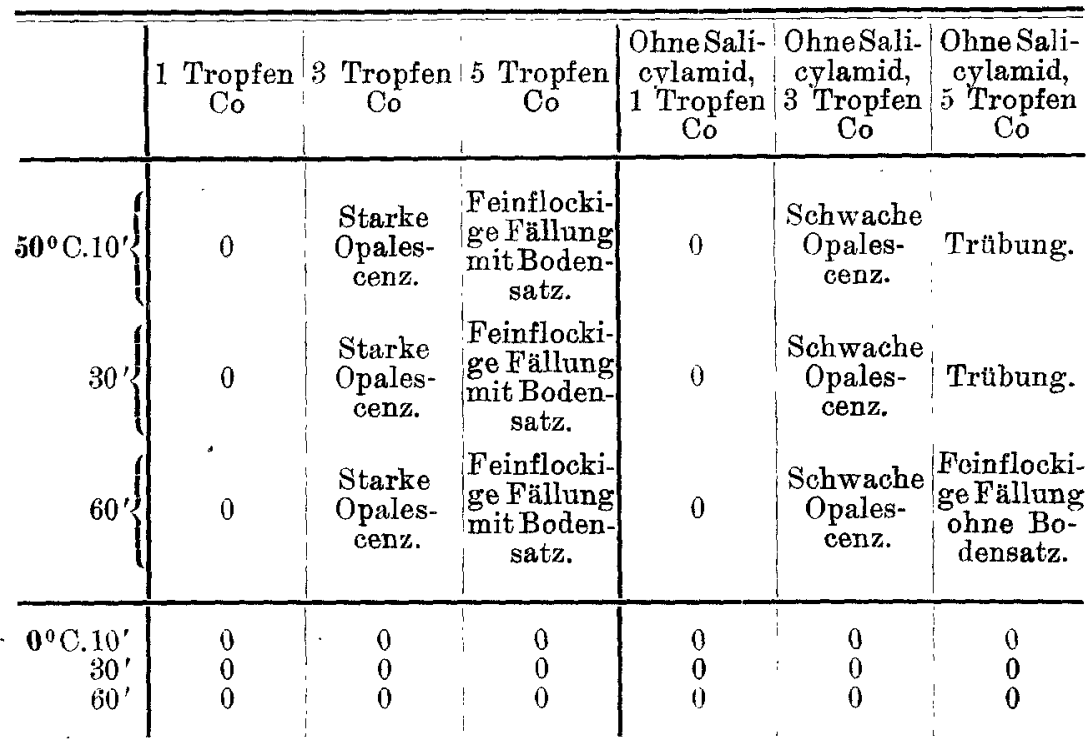

Die Versuche ergeben also übereinstimmend, dass die fünf geprüften Narkotika Isobutylalkohol, Äthylurethan, Propylurethan, Benzamid und Salizylamid die Fällung des Serumalbumins durch Kobaltchlorür bei höherer Temperatur mehr verstärken als bei niederer. Der Schwellenwert der. Narkose liegt für Benzamid und Salicylamid nach H. Meyer bei höherer Temperatur höher als bei niederer.

\section{Der Einfluss der Temperatur anf die Wirkungsstärke der Narkotika.}

Nach den angeführten Ergebnissen hat also die Temperatur keinen deutlichen Einfluss auf die Adsorption der Narkotika an Kohle, und das Flockungsvermögen der Narkotika wird in allen geprüften Fällen. durch Temperaturerhöhung verstärkt. Danach müsste man schliessen, dass in Anbetracht der Angaben von H. Meyer, welche den Ausgangspunkt für diese Untersuchungen bilden, bei der Abwägung zwischen einer Lipoid-, einer Adsorptions- und einer Kolloidtheorie der Narkose die Entscheidung zugunsten der erstgenannten fiele. Bei der naheliegenden Nachprüfung der Narkoseversuche von H. Meyer habe ich mich jedoch vergeblich bemüht, seine Ergebnisse zu reproduzieren.

H. Meyer wählte als Objekt der Narkose Kaulquappen, für welche bei $3^{\circ}$ und bei $30-36^{0}$ die narkotischen Grenzkonzentrationen der 
sechs in der Tabelle Seite 1 angeführten Narkotika aufgesucht wurden. Als besonders demonstrativ bezeichnete Meyer das abwechseInde Einschlafen und Aufwachen der Tiere in ein und derselben Lösung bei entsprechendem Temperaturwechsel und führte als Beispiel dafür an, dass ,in $\frac{\mathrm{m}}{250}$ Chloralhydrat bei $30^{\circ}$ völlig betäubte Tiere einige Zeit nach dem Abkühlen vollständig erwachen, um bei erneutem Erwärmen wiederum in tiefe Narkose zu verfallen ${ }^{6 .}$. Aus diesen Ausführungen muss man schliessen, dass die Kaulquappen, welche mit Benzamid, Salicylamid oder Monacetin in der Kälte narkotisiert wurden, umgekehrt beim Erwärmen wieder erwachen, um in der Kälte abermals gelähmt zu werden.

Demgegenüber habe ich bei der Prüfung von Äthylalkohol, Amylalkohol, Isobutylurethan, Chloralhydrat, Benzamid und Salicylamid nur finden können, dass ihre narkotische Kraft durch Temperaturerhöhung einzig und. allein gesteigert wird.

Meine Versuche wurden bei $0-3^{0}$ und bei $29-30^{\circ}$ im Juni und Juli angestellt. Die Kaulquappen hatten anfangs eine Länge von 18-22, später bis $36 \mathrm{~mm}$; die vier Extremitäten waren schon wohlentwickelt, der Schwanz zum Teil bereits in starker Reduktion begriffen. Die Temperaturänderungen wurden, auch wenn sie innerhalb I Stunde erfolgten, von den Tieren ziemlich gut vertragen. Es befanden sich immer drei bis vier Exemplare in je $200 \mathrm{ccm}$ Narkotikumlösung von verschiedener Konzentration. Da die Kälte die Beweglichkeit der Tiere, wie auch $\mathrm{H}$. Meyer bemerkt, sehr träge macht, so wurde als Kriterium der eingetretenen Narkose nicht das Aufhören spontaner Bewegungen, selbst nicht das Daliegen in Rückenlage angesehen, sondern die Reflexlosigkeit beim Kneifen eines Fusses mit einer feinen Pinzette oder noch besser bei elektrischer Reizung, welche bei unvollständiger Narkose mit einer Bewegung der Extremitäten oder des Schwanzes beantwortet wird. Sämtliche Versuche fanden ihren Abschluss in der Prüfung, ob in Wasser von Zimmertemperatur die Tiere wieder erwachten oder ob die Lähmung in Tod übergegangen war $^{1}$ ).

Beginne ich mit der Schilderung derjenigen Versuche, deren Ausfall von vornherein am beweiskräftigsten erscheint, $d . h$. mit denjenigen Versuchen, bei welchen eine und dieselbe Lösung mit den darin enthaltenen Tieren nacheinander auf verschiedene Temperaturen gebracht wurde, so fand ich folgendes:

1) Die zum Beleg der folgenden Ausführungen dienenden umfangreichen Protokolle wurden beím Druck, wegen Raummangels, fortgelassen. 
1. Tiere, welche in der Wärme unbeweglich geworden waren, blieben auch bei Uthertragung in die Kälte stundenlang unbeweglich; aber es wurde sowohl bei Äthyl- und Amylalkohol als auch bei Benzamid und Salicylamid beobachtet, dass sie danach schliesslich meist wieder beweglich wurden.

2. Tiere, welche in der Kälte unerregbar geworden waren, wurden sehr häufig bei Úbertragung in die Wärme binnen 1/4-2 Minuten wieder beweglich, meist sogar lebhaft beweglich, um dann aber nach 5-15 Minuten von neuem betäubt zu werden. Dies wurde auch bei Benzamid und Salicylamid konstatiert.

Schwieriger ist es, die Schwellenwerte der Narkose bei den verschiedenen Temperaturen festzustellen, weil die Narkose in der Wärme in jedem Fall viel rascher zustandekommt als in der Kälte, und weil die Kälte die Reaktionen so träge macht. Ungefähr liessen sich folgende Werte ermitteln:

$$
\begin{aligned}
& \text { Äthylalkohol . . . . . . . . . . } \frac{0^{\mathrm{m}}}{3} \quad \frac{30^{\circ}}{\mathrm{m}} \\
& \text { Amylalkohol . . . . . . . . } \frac{m}{90} \frac{m}{220} \\
& \text { Isobutylurethan . . . . . . . } \frac{\mathrm{m}}{300} \frac{\mathrm{m}}{600} \\
& \text { Chloralhydrat . . . . . . . . . } \frac{\mathrm{m}}{50} \frac{\mathrm{m}}{165}-\frac{\mathrm{m}}{250} \\
& \text { Benzamid . . . . . . . . . . } \frac{\mathrm{m}}{200} \frac{\mathrm{m}}{500} \\
& \text { Salicylamid . . . . . . . . . } \frac{\mathrm{m}}{600} \frac{\mathrm{m}}{1200}
\end{aligned}
$$

Der Schwellenwert ist also in der Wärme durchweg kleiner als in der Kälte.

Die Versuche, bei denen die Tiere in der gleichen Lösung abwechselnd erwärmt und abgekzuhlt wurden, bedürfen noch einer Erläuterung. Wenn die in der Kälte narkotisierten Tiere zunächst wieder erwachen, um bald darauf von neuem einzuschlafen, so muss man das wohl so deuten, dass die anfangs bestehende Lähmung von der kombinierten Wirkung der Kälte und des Narkotikums herrührt, in dem Sinne, dass weder die Kälte allein noch die Narkotikumkonzentration allein ausreichen würden, die Tiere zu immobilisieren. Dann müssen die Tiere beim Erwärmen zunächst erwachen, bis derjenige Prozess, der der Narkose zugrundeliegt und der durch das Erwärmen verstärkt wird, in genügendem Maasse fortgeschritten ist, um abermals ein 
Einschlafen zú bewirken. Wenn umgekehrt die in der Wärme gelähmten Tiere, in die Kälte übertragen, erst sehr allmählich wieder beweglich werden, so dürfte das daran liegen, dass der in der Wärme abgelaufene Narkoseprozess in der Kälte nur langsam wieder rückgängig gemacht werden kann.

Würde man diese Beobachtungen allein mit Benzamid oder mit Salicylamid machen, dann könnte man bei einer kurzen Versuchsdauer natürlich den Eindruck gewinnen, dass deren narkotische Kraft mit der Erwärmung abnimmt. Aber, wie gesagt, haben wir bei allen sechs Narkoticis, die wir untersuchten, die gleichen Beobachtungen gemacht. Worauf der Widerspruch mit den Befunden von H. Meyer zurückzuführen ist, war zurzeit nicht festzustellen.

\section{Der Einfluss der Temperatur auf die Verteilung der Narkotika zwischen Lebertran und Wasser.}

Begreiflicherweise wurde bei dieser Sachlage nun auch geprüft, ob die Verteilungsversuche von $\mathrm{H}$. Meyer sich reproduzieren liessen. H. Meyer untersuchte die Verteilung der Narkotika bei $3^{0}$ und bei $30^{\circ}$ zwischen gleichen Volumina Olivenöl und Wasser. Zu dem Zweck wurden die Mischungen $11 / 2-2$ Stunden geschüttelt. Dann wurde in der wässerigen Phase das Narkotikum quantitativ teils nach Kjeldahl, teils durch Gefrierpunktmessung bestimmt. Die in der Tabelle Seite I angegebenen Zahlen sind Mittelwerte.

Da mir Olivenöl nicht zur Verfügung stand, so benutzte ich statt dessen Lebertran. Gleiche Volumina davon und von der Narkotikumlösung (je $40 \mathrm{ccm}$ ) wurden bei $0^{0}$ und bei $50^{\circ} 5$ Stunden lang in Flaschen mit Glasstopfen geschüttelt, sofort danach durch 10 Minuten langes Zentrifugieren die Phasen voneinandergetrennt und die wässerige Phase nach Kjeldahl analysiert. Ein gewisser Fehler in den Bestimmungen entsteht wahrscheinlich dadurch, dass trotz des Zentrifugierens an der Grenze von Fett und Wasser eine schmale Zone von emulgiertem Fett bestehen bleibt, in welcher unkontrollierte Mengen von adsorbiertem Narkotikum haften können.

Ich habe mich auf die Untersuchung von Salicylamid und Benzamid beschränkt und dazu noch Isobutylurethan hinzugenommen. Meine Ergebnisse sind die folgenden:

\section{Salicylamid.}

. a) Ausgangslösung . . . . . . . . , 0,239\% nach dem. Schütteln mit Lebertran bei $50^{\circ}$. $0,087 \%$ also Verteilungsquotient bei $50^{\circ} \ldots \ldots$ nach dem Schütteln mit Lebertran bei $0^{0}$. . $0,064 \%$ also Verteilungsquotient bei $0^{0} \ldots \ldots$ 
b) Ausgangslösung . . . . . . . . . . . . 0,245\% nach dem Schütteln mit Lebertran bei $50^{\circ}$. $0,090 \%$

also Verteilungsquotient bei $50^{\circ}$. . . . . 1,72 nach dem Schütteln mit Lebertran bei $0^{\circ}$. . $0,062 \%$ also Verteilungsquotient bei $0^{0} \ldots \ldots . .2,96$

2. Benzamid.

a) Ausgangsiösung . . . . . . . . . . . . 0,810\% nach dem Schütteln mit Lebertran bei $50^{0}$. $0,677 \%$ also Verteilungsquotient bei $50^{\circ} \ldots \ldots$. . 0,20 nach dem Schütteln mit Lebertran bei $0^{0}$. . $0,654 \%$ also Verteilungsquotient bei $0^{0} \ldots \ldots$. . 0,24

b) Ausgangslösung . . . . . . . . . . . . . . 0,800\% nach dem Schütteln mit Lebertran bei $50^{\circ}$. $0,680 \%$ also Verteilungsquotient bei $50^{\circ}$. . . . 0,18 nach dem Schütteln mit Lebertran bei $0^{\circ}$. . $0,642 \%$ also Verteilungsquotient bei $0^{0} \ldots \ldots$. . 0,26

3. Isobutylurethan.

Ausgangslösung . . . . . . . . . . 0,810\% nach dem Schütteln mit Lebertran bei $50^{\circ}$. $0,123 \%$ also Verteilungsquotient bei $50^{\circ} \ldots \ldots$. . 5,58 nach dem Schütteln mit Lebertran bei $0^{0}$. . $0,131 \%$ also Verteilungsquotient bei $0^{0} \ldots \ldots . .5,18$

Die Mittelwerte, mit den Mittelwerten von $\mathrm{H}$. Meyer zusammengestellt, führen also zu folgender Übersicht:

\begin{tabular}{l|c|c|c|c}
\hline \multirow{2}{*}{$\begin{array}{c}\text { Mittlerer Verteilungs- } \\
\text { quotient }\end{array}$} & \multicolumn{2}{|c|}{ für Lebertran } & \multicolumn{2}{c}{ für Olivenöl } \\
\cline { 2 - 5 } & bei $0^{\circ} \mathrm{C}$ & bei $30^{\circ} \mathrm{C}$ & bei $30 \mathrm{C}$ & bei $30-36^{\circ} \mathrm{C}$ \\
\hline Salicylamid. . . . . & 2,84 & 1,74 & 2,23 & 1,40 \\
Benzamid. & 0,25 & 0,19 & 0,67 & 0,44 \\
Isobutylurethan. . . . & 5,18 & 5,58 & - & -
\end{tabular}

Danach ergaben auch meine Versuche, ebenso wie diejenigen von H. Meyer, dass bei Salicylamid und Benzamid der Verteilungsquotient mit steigender Temperatur abnimmt. Dass die Zahlenwerte übereinstimmen, konnte bei der Verschiedenheit der Lösungsmittel von vormherein nicht erwartet werden.

\section{Schlussbetrachtung.}

Den Ausgangspunkt für meine Untersuchungen bildete die Frage, ob der von H. Meyer beobachteten gleichsinnigen Änderung der 
narkotischen Kräfte und der Verteilungsquotienten (Öl:Wasser) bei Änderungen der Temperatur eine entsprechende gleichsinnige Veränderung auch der Adsorbierbarkeit und des Kolloidfällungsvermögens der Narkotika an die Seite zu stellen sei oder nicht, um auf die Weise gegebenenfalls zwischen einer Lipoid-, einer Adsorptions- und einer Kolloidtheorie der Narkose eine Entscheidung zu treffen. Diese Fragestellung hat jedoch im Verlauf der Untersuchung insofern ihre Bedeutung verloren, als unerwartet die Angaben von $\mathrm{H}$. Meyer, nach denen bei manchen Narkoticis Wirksamkeit und Verteilungsfaktor mit der Temperatur steigen, bei anderen mit der Temperatur sinken, nicht bestätigt werden konnte; vielmehr ergab sich, dass unabhängig von der Temperaturvariation der Verteilung die Narkose sich stets mit dem Steigen der Temperatur vertieft. Damit büsst die Lipoidtheorie diejenige Stütze, welche die Temperaturversuche bisher für sie bildeten, ein. Was die Temperaturvariation der Adsorbierbarkeit und des Kolloidfällungsvermögens der Narkotika anlangt, so lehrten die Versuche, dass der Temperaturkoeffizient der Adsorption so geringfügig (und zugleich nicht einsinnig) ist, dass die Beobachtungen nicht gut zugunsten oder zuungunsten der Adsorptionstheorie verwertet werden können. Dagegen geht das Kolloidfällungsvermögen der Narkotika mit ihrer Wirkungsstärke parallel. Dadurch wird aufs neue der Schluss nahegelegt, dass die Narkose eine Dispersionsverminderung der Zellkolloide bedeutet. In eine Diskussion dieser Hypothese soll hier nicht näher eingetreten werden ${ }^{1}$ ); es sei nur in aller Kürze daran erinnert, dass zwar auf der einen Seite zu ihren Ungunsten hervorzuheben ist, dass die bisher genauer untersuchten Ausflockungen von. Kolloiden dureh Narkotika (O. Warburg und Wiesel, Battelli und Stern ${ }^{2}$ ), Kruyt and van Duin ${ }^{3}$ ), Freundlich und Rona ${ }^{4}$, o. Meyerhof ${ }^{5}$ ) Narkotikumkonzentrationen exforderten, welche die den Schwellenwert der Narkose repräsentierenden Konzentrationen erheblich übersteigen, dass aber auf der anderen Seite gute Gründe vorhanden sind, die Verminderung der Zellpermeabilität, welche zu den regelmässigen Vorkommnissen bei der Narkose zu gehören scheint (Osterhout $\left.{ }^{6}\right)$, J. Traube $\left.{ }^{7}\right)$, Arrhenius and Bubanovic $\left.{ }^{8}\right)$,

1) Siehe dazu R. Höber, Physikalische Chemie der Zelle und der GeS. $455 \mathrm{ff}$. 1914. webe. 4. Autl.

2) cf. $i$.

3) Kru yt u. van Duin, Kolloid chem. Beih. S. 269. 1914.

4) $1 . \mathrm{c}$.

5) l. c.

6) Osterhout, Science. Vol. 37, 111. 1913.

7) $1 . \mathrm{c}$.

8) Arrhenius u. Bubanovic, Meddeling, K. Vetensk, Akad. Nobelinstitut 2. Nr. 32. 1913. 
Lillie $^{1}$ ), Joel ${ }^{2}$, H. Winterstein ${ }^{3}$, als einen Kolloidprozess aufzufassen (Höber), und dass dieselben chemischen Mittel, welche in grosser Konzentration durch Kolloidausflockung eine Steigerung der Permeabilität verursachen, in kleiner Konzentration die kolloide Zellmembran verdichten können ${ }^{4}$ ). Wenn aber hier eine Gleichsinnigkeit in der Temperaturabhängigkeit der Narkose und der Kolloidzustandsänderung durch die Narkotika konstatiert wurde, so kann dies bei der Häufigkeit der Verstärkung chemischer und physikochemischer Prozesse durch Temperaturerhöhung nicht als ein besonders schwerwiegendes Argument bei einer Entscheidung über die Natur der der Narkose zugrundeliegenden Vorgänge angesehen werden.

\section{Zusammenfassung.}

1. Die Adsorption der Narkotika Isoamylurethan, Isobutylurethan, Heptylalkohol, Phenylharnstoff, Salicylamid und Benzamid an Tierkohle wird durch Temperaturerhöhung von $0^{\circ}$ auf $50^{\circ}$ nur sehr wenig verändert.

2. Die Ausflockung von Eisenhydroxydsol in Gegenwart von Äthylurethan, Isoamylurethan, Thymol und Benzamid sowie die Ausflockung von denaturiertem Serumalbumin durch Kobaltchlorür in Gegenwart von Isobutylalkohol, Äthylurethan, Propylurethan, Benzamid und Salicylamid werden durch Temperaturerhöhung von $0^{0}$ auf $50^{\circ}$ deutlich verstärkt.

3. Die narkotische Wirkungsstärke von Äthylalkohol, Amylalkohol, Isobutylurethan, Chloralhydrat, Benzamid und Salicylamid steigt bei Erhöhung der Temperatur von $0^{0}$ auf $3^{\circ}$. Dieser Befund für Benzamid und Salicylamid steht in Widerspruch mit Angaben von H. Meyer.

4. Die Verteilung von Benzamid und Salicylamid zwischen Lebertran und Wasser wird durch Steigerung der Temperatur von $0^{0}$ auf $50^{\circ}$, in Ubereinstimmung mit ähnlichen Angaben von H. Meyer, zugunsten des Wassers verschoben.

1) Lillie, Amer. journ. of physiol. 29. 372. 1912.

2) A. Joel, Pflüger's Arch. 161. 5. 1915.

3) Winterstein, Ztschr. f. allg. Phys. 1. 19 (1912) u. 5. 323 (1905) Bioch. Ztschr. 51. 143 (1913) u. 75. 71 (1916).

4) Siehe dazu Höber, Pflüger's Arch. Bd. 166 S. 531, besonders S. 562, $589,600.1917$.

Kie1, 12. Sept. 1918. 